\title{
Use of monoclonal antibodies to recognise osteoclasts in routinely processed bone biopsy specimens
}

\author{
N A Athanasou, B Puddle, J Quinn, C G Woods
}

\begin{abstract}
In decalcified (5\% nitric acid) and undecalcified (glycol-methacrylate or resin embedded) routinely processed bone specimens osteoclasts against resorbing surfaces were identified with monoclonal antibodies directed against leucocyte common antigen (LCA) (PD7/ 26, 2B11), CD68 (KP1), and gpIIIa (Y2/51) but not against HLA-DR (CR3/43 and Ta11B5). Mononuclear cells on resorbing surfaces and occasional mononuclear cells against or near resting surfaces showed a similar pattern of reactivity. This study shows that immunohistochemistry is a sensitive and useful technique for identifying osteoclasts in routinely processed bone specimens. It also suggests a role for mononuclear cells (possibly pre-osteoclasts) in bone resorption.
\end{abstract}

Recognition of osteoclasts in bone biopsy specimens is essential for accurate diagnosis of metabolic bone disease as well as some congenital, inflammatory, and neoplastic lesions of bone. Their identification is facilitated by the use of histochemical markers such as tartrate resistant acid phosphatase ${ }^{1}$ or the vitronectin receptor. ${ }^{2}$ Neither marker is specific for osteoclasts $^{3-5}$ and both require use of fresh or undecalcified material, or fixation, or processing schedules not routinely used in most histopathology laboratories.

The osteoclast antigenic phenotype is now well characterised. Osteoclasts are known to express leucocyte common antigen (LCA), and a restricted set of macrophage antigens. ${ }^{6}$ In this study we have identified several monoclonal antibodies which react with osteoclasts in decalcified and undecalcified bone specimens routinely processed for bone histology.

Nuffield Department of Pathology,

University of Oxford, John Radcliffe Hospital, Headington, Oxford OX3 9DU

N A Athanasou J Quinn

Nuffield Orthopaedic Centre, Oxford N A Athanasou B Puddle

C G Woods

Correspondence to: Dr N A Athanasou Accepted for publication 20 March 1991

\section{Methods}

Material consisted of cases retrieved from the files of the Nuffield Orthopaedic Centre and John Radcliffe Hospital Pathology Departments. These included 15 cases of osteoarthritis of the femoral head fixed for 12-24 hours in $10 \%$ buffered formol saline followed by decalcification overnight in 5\% nitric acid. Blocks taken for histological examination were processed routinely, embedded in paraffin wax, and sectioned at $5 \mu \mathrm{m}$. Ten decalcified and undecalcified bone biopsy specimens examined. Decalcified specimens were proknown to contain osteoclasts were also cessed as above. Undecalcified specimens were embedded in Spurr's resin ${ }^{7}$ and glycol methacrylate $^{8}$ or methyl methacrylate. ${ }^{9}$ In each case formalin fixation was followed by dehydration through graded acetone and water. Infiltration of monomer was achieved using graded resin and acetone followed by two changes of absolute resin. Polymerisation of the methacrylate resins was achieved by the addition of $\mathrm{N}$-N-dimethylaniline at $4^{\circ} \mathrm{C}$. Spurr's resin was polymerised by the addition of S-1 catalyst and incubation at $50^{\circ} \mathrm{C}$ overnight. All sections were cut at $5 \mu \mathrm{m}$ on a Reichert Jung Autocut MK IV and collected on L-polylysine (Sigma) coated slides. Staining of the methacrylate section was carried out with the resin in situ. Spurr's resin was removed before staining using potassium ethoxide with subsequent rinsing in two changes of absolute alcohol.

Monoclonal antibodies used in this study are shown in the table. An indirect immunoperoxidase method was the immunohistochemical procedure used in this study. ${ }^{13}$ Trypsinisation was carried out for 15 minutes before immunohistochemical staining with monoclonal antibodies KP1 and Y2/51. Using this protocol, no immunohistochemical reaction was produced in sections of methylmethacrylate embedded bone biopsy specimens; this indicates that embedding and processing with this resin is not suitable for indirect immunoperoxidase monoclonal antibody staining.

\section{Results}

Several monoclonal antibodies stained antigens known to be present on osteoclasts in fixed, decalcified routinely processed tissues (table). These included PD7/26, 2B11 (both recognising LCA), KP1 (anti-CD68) and Y2/51 (antiCD61) (fig 1). Antibodies against HLA-DR, CR3/43, and Ta11B5 did not stain osteoclasts or other bone cells but reacted with mononuclear cells in the intertrabecular marrow.

Immunoperoxidase staining with the above osteoclast reacting antibodies was highly specific with a membrane reaction noted with antigens against LCA antibodies (fig 1A) and $\mathrm{Y} 2 / 51$, and a cytoplasmic reaction with $\mathrm{KP} 1$ (fig 1B). Osteoblasts, osteocytes, adipocytes and periosteal fibroblasts were unstained. A variable proportion of haemopoietic marrow cells as well as mononuclear inflammatory cells in osteoarthritic subchondral bone cysts reacted with LCA antibodies and KP1. Y2/51 also 
Monoclonal antibodies used in this study and osteoclast reaction

\begin{tabular}{|c|c|c|c|}
\hline Antibody & Reference & $\begin{array}{l}\text { Antigenic } \\
\text { determinants }\end{array}$ & $\begin{array}{l}\text { Osteoclast } \\
\text { reactivity }\end{array}$ \\
\hline $\begin{array}{l}\mathrm{PD} 7 / 26 \\
\text { 2B11 }\end{array}$ & $\begin{array}{l}10 \\
10\end{array}$ & CD45: Leucocyte common antigen & ++ \\
\hline $\begin{array}{l}\text { KP1 } \\
\text { Y2/51 }\end{array}$ & $\begin{array}{l}11 \\
12\end{array}$ & $\begin{array}{l}\text { CD68-like macrophage-associated antigen } \\
\text { CD61-gpIIIa ( } \beta 3 \text { integrin) }\end{array}$ & $\begin{array}{l}++ \\
+\end{array}$ \\
\hline $\begin{array}{l}\text { CR3/43 } \\
\text { Ta11B5 }\end{array}$ & $\begin{array}{l}13 \\
14\end{array}$ & HLA-DR & - \\
\hline
\end{tabular}

++ strong reaction; + weak reaction; - no reaction.

stained some marrow mononuclear cells and strongly stained megakaryocytes and platelets as well as vascular endothelium. In addition to osteoclasts lying in resorption lacunae of cortical or lamellar bone, these antibodies also reacted with mononuclear cells lying against the resorbing bone surface (fig 1). Positively stained mononuclear cells were noted not only in areas of active resorption, in which osteoclasts were also present, but were also found immediately beneath or occasionally among flattened lining cells lining a bone trabecula in areas not undergoing bone resorption (fig 2). In areas of active bone formation, where plump osteoblasts lined the surface of a bone trabecula, these cells were not present. Serial sections were undertaken to determine whether this staining represented a true mononuclear cell or part of a multinucleated osteoclast ${ }^{15}$; this confirmed the presence of true mononuclear cells on these resorbing and resting bone surfaces.

PD7/26, KP1 and Ta11B5 also clearly

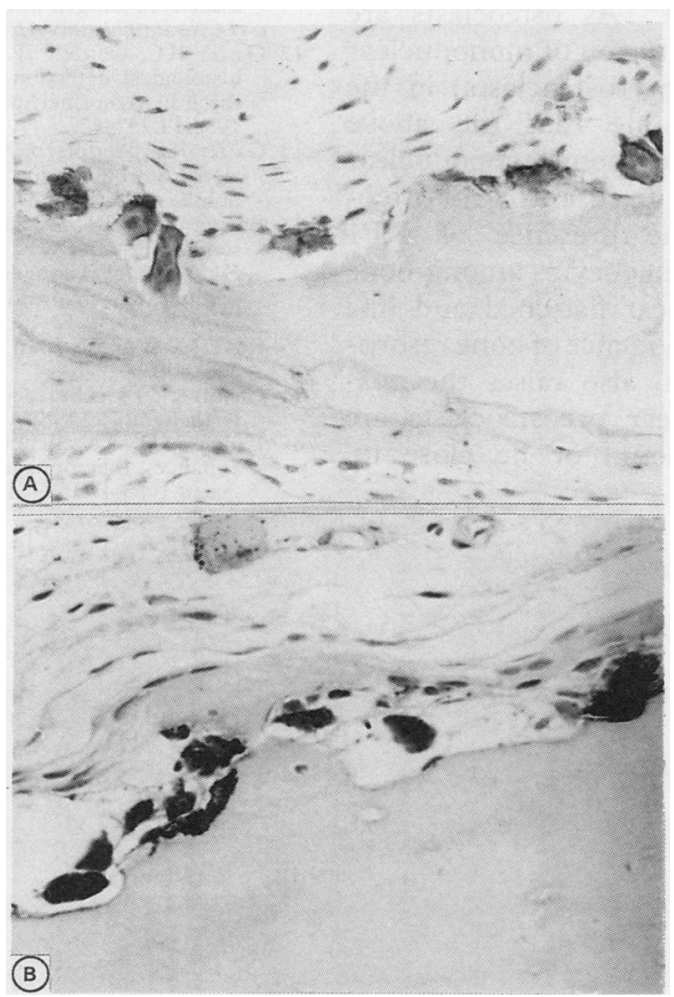

Figure 1 Indirect immunoperoxidase staining of osteoclasts lying against resorbing surfaces of trabecular subchondral bone of osteoarthritic femoral head (decalcified in 5\% nitric acid) showing positive reaction for leucocyte common antigen $(P D 7 / 26)(A)$ and $C D 68$ (KP1) (B). Note no reaction of osteoblasts or osteocytes and positive staining of mononuclear cells in or near resorption lacunae.

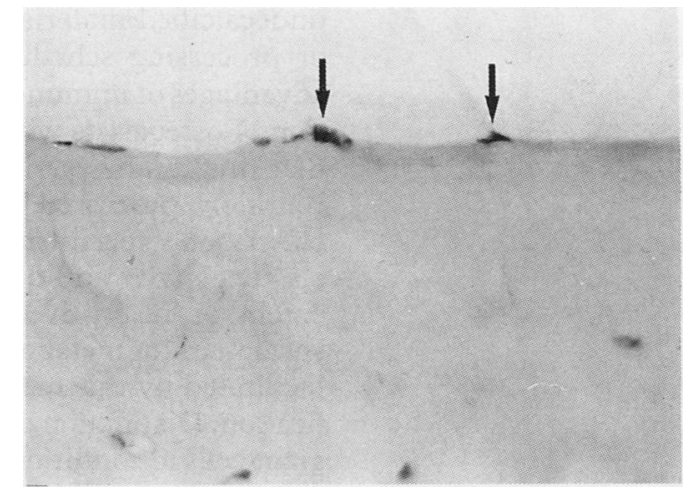

Figure 2 CD68 (KP1) positive mononuclear cells (arrowed) lying against resting trabecular bone surface with flattened lining cells (5\% nitric acid decalcified section).

stained cells in undecalcified glycol-methacrylate and Spurr's resin-embedded bone biopsy specimens (fig 3). The pattern of immunohistochemical staining was similar to that seen in decalcified specimens. KPl and PD7/26 stained osteoclasts and mononuclear cells lying against resorbing surfaces, stromal macrophages, and some haemopoietic elements, while Ta11B5 did not react with osteoclasts or mononuclear cells lying against bone trabeculae.

\section{Discussion}

This study has shown that it is possible to use immunohistochemical techniques to identify osteoclasts by their characteristic antigenic phenotype in routinely processed decalcified and undecalcified specimens submitted for histological examination. Osteoclast expression of LCA, CD68, and gpIIIa was detected using monoclonal antibodies PD7/26, 2B11, KP1 and $Y 2 / 51$ and absence of HLA-DR detected with monoclonal antibodies Ta11B5 and CR3/ 43. Mononuclear cells lying against resorbing surfaces and near resting bone surfaces also showed a similar pattern of antibody staining.

Current markers for the histochemical or immunohistochemical identification of osteoclasts (such as tartrate resistant acid phosphatase, vitronectin receptor) require the use of

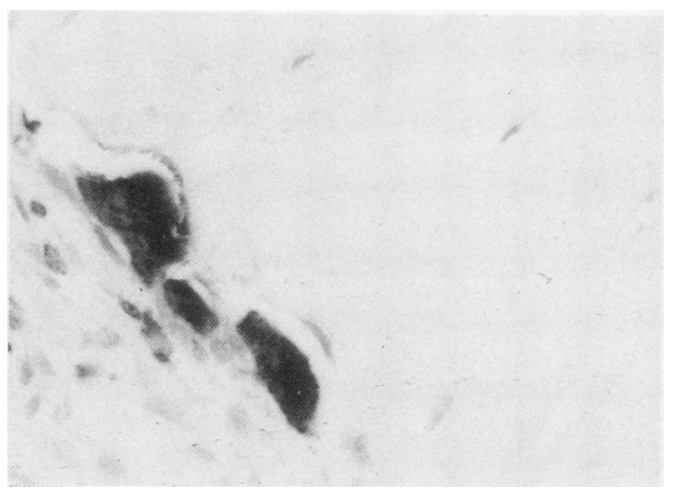

Figure 3 Indirect immunoperoxidase staining of undecalcified glycol methacrylate embedded bone biopsy specimen of hyperparathyroid bone disease showing osteoclasts lying against resorbing surface; these show a positive cytoplasmic reaction for CD68 (KP1). 
undecalcified material and specialised fixation or processing schedules. ${ }^{12}$ One of the major advantages of immunohistochemical identification of osteoclasts with the above antibodies is that this can be carried out on formalin fixed, routinely processed decalcified and undecalcified bone specimens. Recognition of osteoclastic activity and quantitation of the area or extent or resorptive activity in bone biopsy specimens for metabolic bone disease should be facilitated by this method of osteoclast identification. Distinction of osteoclasts from tumour giant cells in conditions such as osteosarcoma is also made possible by this technique, particularly as specialised fixatives are not routinely used for processing of biopsy specimens or specimens of bone tumours. These antibodies identifying leucocytes or macrophage antigens are also useful for determining the nature of both mononuclear and multinucleated cells in highly cellular, inflammatory, vascular or neoplastic lesions of bone where it may occasionally be difficult to distinguish between stromal and reactive cells.

Mononuclear cells with a similar antigenic phenotype to that of osteoclasts (HLA-DR negative, LCA positive, CD68, gpIIIa positive) were found against active trabecular resorption surfaces as well as scattered among flattened mononuclear cells lining resting trabecular surfaces. This suggests that mononuclear cells also participate in bone resorption. Although osteoclasts are known to be the principal cell involved in bone resorption, a role for mononuclear cells in the resorptive process, particularly macrophage-like cells, has often been proposed. ${ }^{16} 17$ As osteoclasts are known to be formed by fusion of mononuclear osteoclast precursors (pre-osteoclasts) in the bone, it is also possible that the above antibodies are identifying some mononuclear cells which are destined to fuse to form osteoclast polykaryons. The presence of KP1 positive mononuclear phagocytes among bone lining cells, which appear flattened and line trabeculae showing no evidence of bone resorption or bone formation, also raises the possibility that mononuclear preosteoclasts are normally scattered among, or lie close to, resting bone surfaces.
We thank Miss L Watts for typing the manuscript and Dr K Pulford and Dr D Y Mason for kindly supplying the antibodies used in this study. We also acknowledge the advice and technical help of Professor S L Teitelbaum and Professor M D Fallon. NAA was an Arthritis and Rheumatism Council Fellow in Pathology.

1 Minkin C. Bone acid phosphatase: tartrate-resistant acid phosphatase as a marker of osteoclast function. Calcif Tissue Int 1982;34:285-90.

2 Horton MA, Lewis D, McNulty K, Pringle JAS, Chambers TJ. Monoclonal antibodies to osteoclastomas (giant cell bone tumours): definition of osteoclast-specific antigens. Cancer Res 1985;45:5663-9.

3 Hattersley G, Chambers TJ. Generation of osteoclastic function in mouse bone marrow cultures: multinuclearity and tartrate-resistant acid phosphatase are unreliable markers for osteoclastic differentiation. Endocrinology 1989;124:1689-96.

4 Bianco P, Ballanti P, Bonucci E. Tartrate resistant acid phosphatase in bone forming cells. Calcif Tissue Int phosphatase in bon

5 Athanasou NA, Quinn J, Horton MA, McGee J O'D. New sthanasou NA, Quinn J, Horton MA, McGee J O'D. New
sites of cellular vitronectin receptor immunoreactivity detected with osteoclast-reacting monoclonal antibodies $13 \mathrm{C} 2$ and 23C6. Bone Mineral 1990;8:7-22.

6 Athanasou NA, Quinn J. Immunophenotypic differences between osteoclasts and macrophage polykaryons: immunohistological distinction and implications for osteoclast ontogeny and function. J Clin Pathol 1990; 43:997-1004.

7 Edwards CW, Krypczyk A, Brownhill A. Plastic embedding of transbronchial biopsy specimens for light microscopy. J Clin Pathol 1979;32:1294-8.

8 Baron R, Vignery A, Neff L, Silvergate A, Santa Maria A. Processing of undecalcified bone specimens for bone Processing of undecalcified bone specimens for bone histomorphometry. In: Recker RR, ed. Bone histomorphometry techniques and

9 Hott M, Marie PJ. Glycol methacrylate as an embedding medium for bone. Stain Technol 1987;62:51-7.

10 Warnke RA, Gatter KC, Falini B, et al. Diagnosis of human lymphoma with monoclonal anti-leukocyte antibodies. N Engl J Med 1983;309:1275-81.

11 Pulford KAF, Rigney EM, Micklem KJ, et al. KP1: a new monoclonal antibody that detects a monocyte/macrophage associated antigen in routinely processed tissue sections. $J$ Clin Pathol 1989;42:414-21.

12 Gatter KC, Cordell JL, Turley H, et al. The immunohistological detection of platelets, megakaryocytes and thrombi in routinely processed specimens. Histopathology thrombi in routin

13 Gatter KC, Abdulaziz Z, Beverley P, et al. Use of monoclonal antibodies for the histopathological diagnosis of clonal antibodies for the histopathological diagnosis

14 Epenetos AA, Bobrow LG, Adams TE, Collins CM, Isaacson PG, Bodmer WF. A monoclonal antibody that detects HLA-D region antigen in routinely fixed, wax embedded sections of normal and neoplastic lymphoid tissues. J Clin Pathol 1985;38:12-17.

15 Kaye M. When is it an osteoclast? J Clin Pathol 1984;37: 398-400.

16 Chambers TJ. The cellular basis of bone resorption. Clin Orthop Rel Res 1980;151:283-93.

17 Fallon MD, Teitelbaum SL, Kahn AJ. Multinucleation enhances macrophage-mediated bone resorption. Lab Invest 1983;49:159-64. 